\title{
Vancomycin in peritoneal dialysis: Clinical pharmacology considerations in therapy.
}

\author{
Edwin Lam \\ Thomas Jefferson University \\ Yi Ting Kayla Lien \\ University of Florida \\ Walter K. Kraft \\ Thomas Jefferson University \\ Beth Piraino \\ University of Pittsburgh \\ Follow this and additional works at: https://jdc.jefferson.edu/petfp
Valvanera Vozmediano \\ iveastityfaKelloridalacal Pharmacology Commons \\ Let us know how access to this document benefits you
}

See next page for additional authors

\section{Recommended Citation}

Lam, Edwin; Lien, Yi Ting Kayla; Kraft, Walter K.; Piraino, Beth; Vozmediano, Valvanera; Schmidt, Stephan; and Zhang, Jingjing, "Vancomycin in peritoneal dialysis: Clinical pharmacology considerations in therapy." (2020). Department of Pharmacology and Experimental Therapeutics Faculty Papers. Paper 118.

https://jdc.jefferson.edu/petfp/118

This Article is brought to you for free and open access by the Jefferson Digital Commons. The Jefferson Digital Commons is a service of Thomas Jefferson University's Center for Teaching and Learning (CTL). The Commons is a showcase for Jefferson books and journals, peer-reviewed scholarly publications, unique historical collections from the University archives, and teaching tools. The Jefferson Digital Commons allows researchers and interested readers anywhere in the world to learn about and keep up to date with Jefferson scholarship. This article has been accepted for inclusion in Department of Pharmacology and Experimental Therapeutics Faculty Papers by an authorized administrator of the Jefferson Digital Commons. For more information, please contact: JeffersonDigitalCommons@jefferson.edu. 


\section{Authors}

Edwin Lam, Yi Ting Kayla Lien, Walter K. Kraft, Beth Piraino, Valvanera Vozmediano, Stephan Schmidt, and Jingjing Zhang 
Running title: Vancomycin in PD

Authors and Affiliations: Edwin Lam ${ }^{1}$, Yi Ting (Kayla) Lien ${ }^{2}$, Walter K. Kraft ${ }^{1}$, Beth Piraino ${ }^{3}$, Valvanera Vozmediano ${ }^{2}$, Stephan Schmidt ${ }^{2}$, Jingjing Zhang ${ }^{4}$

Department of Pharmacology and Experimental Therapeutics, Thomas Jefferson University, Philadelphia, Pennsylvania, USA ${ }^{1}$; Center for Pharmacometrics and Systems Pharmacology, Department of Pharmaceutics, College of Pharmacy, University of Florida, Lake Nona (Orlando), Florida, USA ${ }^{2}$; Renal Electrolyte Division, University of Pittsburgh School of Medicine, Pittsburgh, Pennsylvania, USA ${ }^{3}$; Department of Medicine, Division of Nephrology, Thomas Jefferson University, Philadelphia, PA, USA ${ }^{4}$

Acknowledgements: Edwin Lam is supported by a National Institutes of Health institutional (NIH) training grant T32GM008562

\section{Corresponding author:}

Jingjing Zhang, MD, PhD; Jingjing.zhang@jefferson.edu

Department of Medicine - Division of Nephrology

Thomas Jefferson University 833 Chestnut East, Suite 700

Philadelphia, PA 19107-5244

Tel: 215-955-6550

Fax: 215-503-4099

\section{Manuscript Metrics}

Title Characters (with spaces) $\quad 82 / 90$

Running Title Characters (with spaces) $16 / 30$

Body of manuscript (words) 3465/4000

Figures 2

Tables 4 
50 Intraperitoneal vancomycin is the first line therapy in the management of peritoneal dialysis-related

51 peritonitis. However, due to the paucity of data, vancomycin dosing for peritonitis in patients on

52 automated peritoneal dialysis (APD) is empiric and based on clinical experience rather than evidence.

53 Studies in continuous ambulatory peritoneal dialysis (CAPD) patients have been used to provide

54 guidelines for dosing and are often extrapolated for APD use, but it is unclear if this is appropriate. This

55 review summarizes the available pharmacokinetic data used to inform optimal dosing in patients on

56 CAPD or APD. The determinants of vancomycin disposition and pharmacodynamic effects are critically

57 summarized, knowledge gaps explored, and a vancomycin dosing algorithm in peritoneal dialysis

58 patients is proposed.

59

60

61

62

63

64

65

66

67

68

69

70

71 Key words: Automated peritoneal dialysis; continuous ambulatory peritoneal dialysis; anuria; residual

72 kidney function; peritonitis; pharmacokinetics; pharmacodynamics. 


\section{INTRODUCTION}

Vancomycin is often selected as empiric first line therapy for suspected Gram-positive organisms in peritoneal dialysis (PD) related peritonitis. However, data on vancomycin dosing in various PD modalities are limited, especially for automated peritoneal dialysis (APD). The paucity of well-designed pharmacokinetic studies has led to vancomycin dosing guidelines for PD patients that are based on limited information resulting in the possibility of achieving sub-or supra-therapeutic trough concentrations in this special patient population.(1)

\section{PRINCIPLES OF VANCOMYCIN THERAPY}

Vancomycin is a tricyclic glycopeptide antibiotic with broad spectrum activity against Grampositive bacteria. It is effective for the treatment of Gram-positive infections including peritonitis and is the drug of choice for methicillin-resistant Staphylococcus aureus (MRSA). Vancomycin is poorly absorbed following oral administration. Therefore, it is commonly administered as an intravenous infusion, except in peritoneal dialysis where the route is preferentially intraperitoneal. Approximately $50 \%$ of vancomycin is protein-bound in plasma with a variable volume of distribution ranging between 0.4-1 L/kg in the non-PD population. $(2,3)$ An initial distribution half-life ranging from 30 minutes to 1 hour followed by a mean terminal elimination half-life ranging from 6-12 hours were determined following intravenous dosing in patients with normal renal function.(3) Metabolism is negligible and elimination occurs primarily through glomerular filtration, such that advanced renal disease substantially reduces the clearance of vancomycin resulting in an elimination half-life of about 7.5 days compared to 4-6 hours in normal patients. This means that in patients with kidney failure, the dosing of vancomycin must be adjusted. $(4,5)$

The Clinical and Laboratory Standards Institute (CLSI) has established the vancomycin breakpoint for susceptible $S$. aureus isolates with MIC values of $\leq 2 \mathrm{mg} / \mathrm{L}$ and intermediate or resistant 
97 for MIC values greater than $2 \mathrm{mg} / \mathrm{L}$.(6) Despite the CLSI defined breakpoints, treatment failure for

98 patients infected with S. aureus and vancomycin MICs between 1-2 mg/L have been reported compared

99 to those with lower reported MICs. $(7,8)$ This may be due to inappropriate selection of doses that are

100 sufficiently high to maintain plasma concentrations that exceed the MIC.

101 To optimize the vancomycin exposure-response relationship for efficacy during S. aureus

102 infections, one must examine the ratio of the area under the concentration-time curve and the MIC

103 (AUC/MIC). Vancomycin trough concentrations between $15-20 \mathrm{mg} / \mathrm{L}$ for MIC breakpoints $\leq 1 \mathrm{mg} / \mathrm{L}$

104 ensures a ratio of $\geq 400$ and has been an advocated target for clinical effectiveness. $(3,9)$ It should be

105 noted that goal trough values recommended by consensus guidelines for efficacy may lead to

106 nephrotoxicity, which might be a consideration for patients on PD with residual kidney function.(10) This

107 however, is not well studied. In practice, clinical judgement together with therapeutic drug monitoring

108 (TDM) of steady-state vancomycin plasma concentrations is a common approach in the treatment of

109 peritonitis in PD.

110

PHARMACOKINETIC/PHARMACODYNAMIC MODELING AND SIMULATION

112

Pharmacokinetic/pharmacodynamic modeling and simulation is an innovative approach that can

113 help inform crucial decisions, such as predicting clinical endpoints of new doses and dosing regimens or

114 optimization of drug regimens. By understanding what the body does to the drug (Pharmacokinetics)

115 and what the drug does to the body (Pharmacodynamics), dosing regimens can be tailored to the PD

116 population to avoid nephrotoxicity, retain antimicrobial eradication and suppressing the emergence of

117 resistance. Regulatory authorities mandate the submission of pharmacokinetic/pharmacodynamic

118 evaluations for drug application, which include dose evaluation in special populations. However, despite

119 the evaluation of the need of dose adjustments for patients with end stage renal disease (ESRD) - such 
as those on hemodialysis- the process is not well established for old drugs. Even in those cases when dose adjustments are proposed for patients with ESRD, there is minimal attention in patients on PD. This review aims to summarize the available evidence on vancomycin pharmacokinetic and pharmacodynamic PD-related studies, address the physicochemical and PD modality-specific considerations- with attention on APD, and highlight areas where research is needed on dosing vancomycin for PD-related peritonitis.

\section{VANCOMYCIN PHYSICOCHEMICAL PROPERTIES AND DRUG TRANSPORT ACROSS THE PERITONEUM}

Movement of vancomycin from the peritoneum cavity to plasma is based on Fick's Law (figure 1). Middle molecular weight solutes such as vancomycin $(1,486 \mathrm{~g} / \mathrm{mol})$ are dependent on dwell time during PD for absorption into the plasma. Based upon a single dose study of six non-infected subjects on PD, vancomycin has a lower dialysate to plasma ratio than urea and creatinine at two hours.(11) There is no correlation between vancomycin PD clearance and dialysis adequacy (Kt/V) following an intravenous dose in patients on APD.(12)

Teicoplanin, a glycopeptide antibiotic with a similar molecular structure $(1,564 \mathrm{~g} / \mathrm{mol})$ and spectrum of activity to vancomycin, was studied in non-infected adults on continuous ambulatory peritoneal dialysis (CAPD).(13) The absolute bioavailability $\left(F_{i p}\right)$ was calculated using dialysate drug concentration (corrected for amount remaining in the cavity) and drug amount sampled, which was then plotted against a total dwell time of five hours. Teicoplanin systemic bioavailability, reflecting transfer from the peritoneal space, was directly related to dwell time. Furthermore, the consistency in absorption increased with time suggesting that complete and less variable bioavailability with teicoplanin can be achieved with longer dwell times. 
143 membrane. Vancomycin intraperitoneal to systemic transfer rate increases in patients with

144 inflammatory peritonitis.(14)

145
VANCOMYCIN BIOAVAILABILITY DURING CONTINUOUS AMBULATORY PERITONEAL DIALYSIS Vancomycin pharmacokinetics has primarily been studied in patients on CAPD. Bioavailability vancomycin reaching systemic circulation from the peritoneal space relative to an intravenous dose, is approximately $50 \%$.(15) Supporting the hypothesis of a leaky peritoneum due to membrane inflammation, patients on CAPD with peritonitis have a $\mathrm{F}_{\text {ip }}$ of $70-91 \% .(14,16)$ Bioavailability changes can also be observed with different age cohorts. For example, in a pediatric study in children aged 5-17 years old, the bioavailability was reported to be as high as about $70 \%$ in the absence of peritonitis.(17)

A summary of the absorption parameters from studies conducted in infected and non-infected patients on CAPD is depicted in table 1. The equilibration half-life describes the time allowed for drug transfer between the peritoneal space to the systemic circulation following an intraperitoneal dose of vancomycin. Following intraperitoneal dosing, vancomycin equilibration half-life in patients on CAPD without peritonitis was 2.9 hours and those with peritonitis 1.6-2.9 hours.(18-20) Assuming no differences between peritoneum transport in those with or without peritonitis and five half-lives, steady-state equilibrium between the dialytic compartment and systemic circulation would be achieved following a 10-15 hour dwell.

\section{VANCOMYCIN BIOAVAILABILITY DURING AUTOMATED PERITONEAL DIALYSIS}

Vancomycin possess the desired physiochemical properties as a drug candidate for intraperitoneal administration in APD patients. In addition, with its well-established stability in PD fluids, 
bioavailability is adequate as long as sufficient dwelling time is allowed for drug absorption. However,

167 the appropriate duration of the dwell time has not been well studied. Hence, it is crucial to monitor vancomycin levels frequently to adjust dosing to get therapeutic concentrations in each individual patient.

\section{VANCOMYCIN CLEARANCE DURING PERITONEAL DIALYSIS}

173 clearance. Total body clearance is the sum of clearances contributed from elimination organs, mainly 174 kidneys, in the case of vancomycin, and is defined as the volume of plasma cleared of vancomycin per 175 time unit. Elimination processes in PD patients include those originating from residual kidney function 176 (RKF), other non-renal sources plus the drug cleared through PD. Total body clearance is especially

177 important as it controls the overall exposure of vancomycin for the given bioavailability achieved from a 178 dwell. Dialytic clearance is defined as the volume of plasma that has been cleared of vancomycin (i.e. 179 removed from systemic circulation into the peritoneal space) by PD per unit time. Figure 1 describes the 180 various clearance processes involved in vancomycin elimination following an intraperitoneal dose.

181 Moreover, a summary of vancomycin pharmacokinetic systemic parameters is provided in table 2.

182 Vancomycin clearance in patients on PD differs among studies due to several factors including the 183 presence or absence of peritonitis, presence and extent of RKF, dwell times, dialysate volume, effect of 184 antibiotic-free PD exchanges, and age.(21) may not be sufficient to reach equilibration between the dialysate and plasma. Studies in non-infected adult CAPD patients report dialytic clearances ranging between $1.2-2.4 \mathrm{~mL} / \mathrm{min}$, which account for 20- 
$25 \%$ of the total plasma clearance. $(15,22,23)$ In patients with peritonitis, vancomycin dialytic clearance increases to $3.8 \mathrm{~mL} / \mathrm{min}$ following a less-than five-hour exchange.(24) Clearances of up to $8.5 \mathrm{~mL} / \mathrm{min}$ after the first 4 hours of exchange have also been reported.(16) Vancomycin clearance through elimination from the drained peritoneal dialysate contributes to $20-70 \%$ of the total plasma clearance. $(16,24)$ As a consequence, vancomycin elimination half-life in the systemic circulation ranges between 66-115 hours in patients on CAPD. $(22,24-26)$ One major reason in the reported variability in the plasma half-life could be the difference in the sampling times which may not completely capture the decline of the plasma concentrations during the terminal elimination phase. Table 2 also includes a summary of above parameters in these patients.

\section{AUTOMATED PERITONEAL DIALYSIS}

Studies conducted in the APD population are only reserved to the parenteral administration of antibiotics in patients without peritonitis, yet vancomycin is primarily used to treat peritonitis and is mostly administered intraperitoneally. $(27,28)$ With rapid cycling, the dialytic clearance of vancomycin may be increased. Therefore, if doses and dwell times used for those on the cycler are similar to those in CAPD, the result may be sub-therapeutic levels due to frequent exchanges.

To date, there has only been one study exploring intravenous vancomycin disposition in subjects on APD.(12) The primary objective was to characterize vancomycin pharmacokinetic parameters in adults without peritonitis after a single intravenous dose. Following the intravenous administration of 15 $\mathrm{mg} / \mathrm{kg}$, subjects received three cycle treatments over the course of eight hours followed by two 8-hour off-cycler dwells for a total of 24 hours. A 2-liter $2.5 \%$ dextrose dialysate prescription was used during and off-cycler dwell. The plasma half-life was 11.6 hours following an on-cycler exchange consisting of three 2-hour dwells. When the same patients were removed from the cycler and allowed to dwell for 78 hours, the plasma half-life increased to 62.8 hours. Although vancomycin was not dosed 
214 intraperitoneally in this study, rapid decline in the plasma half-life support the contribution of APD in the

215 removal of drug. Clearance values did not largely differ from those on CAPD. Approximately 30\% of

216 vancomycin was removed by APD relative to the total plasma clearance, which is close to the proportion

217 reported in patients on CAPD. Although intraperitoneal vancomycin administration is recommended by

218 guidelines in patients with PD peritonitis, this intravenous administration study provides a valuable

219 insight towards drug clearance during APD.(29) It should be noted that intravenous administration of

220 vancomycin may not be adequate to achieve effective antibacterial concentrations in the

221 peritoneum.(30)

222 The current International Society for Peritoneal Dialysis (ISPD) guideline recommends

223 supplemental dosing in order to achieve plasma vancomycin troughs above $15 \mathrm{mg} / \mathrm{L}$ when administered

224 intermittently. Alternatively, temporarily switching to CAPD is another option for APD patients who

225 develop peritonitis, but is not always feasible. In patients on APD, leveraging the long dwell to

226 appreciate optimal vancomycin transfer is appropriate to ensure adequate time to achieve and sustain

227 therapeutic levels.

228

229

IMPACT OF RESIDUAL KIDNEY FUNCTION (RKF) AND TREATMENT OUTCOME

Residual kidney function in PD patients will have a profound effect for hydrophilic drugs

231 removed exclusively through renal filtration. Enhanced drug clearance from RKF may have implications

232 to treatment outcomes in patients with PD-related peritonitis. Therefore, patients with greater RKF may

233 require higher or more frequent antibiotic dosing.

234 The importance of RKF on the outcome of PD-related peritonitis in patients treated with

235 antibiotics has been discussed for more than ten years, but the data describing this relationship are still

236 scarce and controversial. The ISPD 2010 update on PD-related infections has previously recommended a

$23725 \%$ increase in antibiotic dose in patients with a daily urine output of over $100 \mathrm{~mL} .(31)$ This 
recommendation has been removed in the updated 2016 guideline, which reflects the lack of evidence

239 to support this empiric recommendation.(29) In a retrospective study examining the impact of RKF on

240 vancomycin concentrations, the influence of RKF was found to not have a significant impact.(32)

241 Vancomycin concentrations appeared lower in patients who were non-anuric across both modalities

242 even though a $25 \%$ higher dose was administered to those with RKF. This however was concluded to not

243 be statistically significant. Similar results have been published showing no difference in treatment

244 outcomes in non-anuric and anuric patients treated with cefazolin and gentamicin.(33)

In contrast, a recent study investigating the relationship between RKF and PD-related peritonitis

247 function.(34) Treatment failure in those with Gram-positive and culture-negative peritonitis were found

248 to be significantly higher for patients with a urinary creatinine clearance greater than $0-5 \mathrm{~mL} / \mathrm{min}$

249 compared to those who were anuric. Significantly higher relapse and recurrence were observed in those

250 patients with Gram-positive or culture-negative infections and creatinine clearances greater than 5

$251 \mathrm{~mL} / \mathrm{min}$. Cefazolin and vancomycin were the main antibiotics used in the study. These observations may

252 be useful when attempting to understand the impact of RKF on treatment outcomes and raise the

253 question as to whether patients with RKF greater than $5 \mathrm{~mL} / \mathrm{min}$ were under-dosed with antibiotic in

254 previous studies.

In patients treated with vancomycin, RKF may account for $10-23 \%$ of the total body clearance in

256 PD. $(12,22)$ Studies examining the impact of RKF on vancomycin clearance, exposure, and treatment

257 outcomes in PD-related peritonitis are limited. Interestingly, for the subset of patients with a glomerular

258 filtration rate greater than $5 \mathrm{~mL} / \mathrm{min}$, RKF accounted for $39-84 \%$ of the total vancomycin clearance.(12)

259 It would appear that the impact from RKF has a substantial effect on the total clearance of vancomycin.

260 Thus, the recent 2016 ISPD recommendation of removing the $25 \%$ dosage increase to account for RKF is

261 unclear as most of the studies cited accounted for a dosage increase for those who were non-anuric.(32, 
35) In the absence of additional studies, dosage adjustments to account for RKF may still be appropriate as there is a substantial contribution observed on the total vancomycin clearance. For now, we can only speculate that the resulting impact in treatment failure for Gram-positive peritonitis may be associated with higher drug clearance values in patients with creatinine clearances greater than $5 \mathrm{~mL} / \mathrm{min}$.

\section{THERAPEUTIC DRUG MONITORING AND PHARMACODYNAMIC RESPONSE}

Vancomycin therapeutic drug monitoring is critical for patients with peritonitis and is routinely

performed because 1) the concentration plays the key component for the effect and 2) the initial

antibiotic dose is needed to target the maximum effect in order to allow proper eradication and

271 prevention of resistance. Moreover, the treatment window timeframe is crucial for patients. Hence,

272 appropriate plasma sampling during this timeframe is important, but may be difficult as the turnaround

273 time for assay results is a rate-limiting factor in achieving desired therapeutic drug levels. Furthermore,

274 not only is it important to ensure that the initial dose is sufficient, but also if that initial dose is able to

275 maintain therapeutic effect throughout treatment. Yet, current clinical practice is based on empirical

276 decisions, which may not reflect the most optimized regimen for patients on PD.

The traditional role of plasma trough concentration monitoring has been conflicting in the PD

278 population. Unlike the established optimal plasma trough levels of $10-15 \mathrm{mg} / \mathrm{L}$ for uncomplicated

279 infections or $15-20 \mathrm{mg} / \mathrm{L}$ for complicated infections, there is substantial interpatient variability for those

280 patients on PD. Higher rates of PD-related peritonitis relapse have been associated with a cumulative 4-

281 week plasma trough below $12 \mathrm{mg} / \mathrm{L}$ when compared to those maintained above that threshold.(36) In

282 this study, vancomycin was given intravenously where plasma levels were maintained above $12 \mathrm{mg} / \mathrm{L}$

283 rather than the current $15 \mathrm{mg} / \mathrm{L}$ recommendation by the ISPD. The type of modality did not differ

284 among the outcome groups, however vancomycin clearance and RKF information were not reported

285 which may have contributed to variability in the plasma concentration. On the other hand, data from a 
single-center study involving 34 PD patients experiencing PD-related peritonitis showed no relationship

287 between plasma vancomycin levels measured during the first week and PD-related peritonitis

288 outcomes.(37) Here, CAPD was reportedly the most frequent modality (80\%) used with an average

289 residual creatinine clearance of $2.8 \mathrm{~mL} / \mathrm{min} / 1.73 \mathrm{~m}^{2}$. Vancomycin was dosed based on ISPD

290 recommendations and plasma levels were maintained above $15 \mathrm{mg} / \mathrm{L}$. Of these 34 PD patients with

291 confirmed Gram-positive infections, 43\% of cases were associated with coagulase-negative

292 Staphylococcus ssp. while only $11 \%$ of cases were due to MRSA. In total, although the frequency and

293 level of vancomycin measurement was not associated with adverse clinical events during the first week

294 of treatment, the number of patients studied may be too small to draw a firm conclusion.

295 Pharmacokinetic sources of variability can be explained in part due to varying exchanges provided by the

296 patient's PD modality, impact from RKF, and peritoneum physiology affecting drug absorption. In

297 addition, the pharmacodynamics- or bacterial susceptibility measured by its MIC-contributes to the

298 variability in clinical response, which may not be explained due to vancomycin pharmacokinetics alone.

299 Taken together, vancomycin shows substantial interindividual variability in clinical response for

300 patients treated for PD-related peritonitis. Table 3 gives an overview of the

301 pharmacokinetic/pharmacodynamic factors to be considered at the time of TDM of vancomycin in

302 patients on both CAPD and APD regimens.

\section{CONSIDERATIONS FOR INTRAPERITONEAL DOSING}

Clinicians should consider dwell times that achieve substantial equilibrium between the

peritoneum compartment and the systemic circulation. The reported bioavailabilities in literature are

307 dwell-time specific and may not be applicable in all patient-specific situations. Therefore, considering

308 the transfer half-life between the dialytic compartment and systemic circulation can be useful to

309 understand the time that it takes to reach equilibrium (i.e., steady-state). This may take up to 15 hours 
considering a transfer half-life of 3 hours.(19) In this situation, dosing during the long-dwell interval may

311 provide adequate drug absorption to achieve therapeutic concentrations in plasma in patients on APD.

The bioavailability of vancomycin significantly increases during PD-related peritonitis. Plasma

314 intraperitoneal doses of vancomycin in PD-related peritonitis.(14, 16) Alternatively, plasma

315 concentrations as low as $10 \mathrm{mg} / \mathrm{L}$ have been reported following a 6 hour dwell using a $500 \mathrm{mg}$

316 intraperitoneal dose in PD-related peritonitis.(38) Regardless of the PD modality, absorption does not

317 largely change between CAPD or APD based on the equilibration half-lives reported.(12, 19, 20)

In patients with PD peritonitis on APD, doses of $15-20 \mathrm{mg} / \mathrm{kg}$ together with dwell times ranging

from 10-15 hours may be more appropriate than the targeted concentration strategy mentioned above.

TDM should also be performed to evaluate therapeutic and toxic concentration fluctuations and to maintain concentrations above $15 \mathrm{mg} / \mathrm{L}$ as recommended by the ISPD guidelines.

\section{FUTURE RESEARCH AND DOSING GUIDELINES IN AUTOMATED PERITONEAL DIALYSIS}

Empiric Gram-positive management using vancomycin for PD-related peritonitis in patients on

325 APD is summarized in figure 2. This algorithm accounts for RKF and suggests a dosage increase of $20 \%$

326 for those who are non-anuric with a creatinine clearance greater than $5 \mathrm{~mL} / \mathrm{min}$ based on observational

327 outcome studies.(34) In addition, monitoring plasma vancomycin concentrations 48 hours post-dose

328 would be appropriate based on previous experience. As such, re-dosing would be necessary to maintain

329 the targeted $15 \mathrm{mg} / \mathrm{L}$ concentration. During this time, adjustments to antibiotic therapy should be

330 guided by the microbiology or susceptibility report. This should be practiced together with routine TDM

331 at appropriate sampling times to rationally select the effective dose for each patient. Pharmacometric

332 modeling and simulation could help to increase the knowledge on vancomycin dose exposure response

333 relationship and propose optimal dosing and TDM strategies in PD patients. 
As above recommendations are based on limited evidence, dedicated studies are needed to

support them. Table 4 highlights the knowledge gaps and propose future research topics to better tailor vancomycin treatments in PD patients with peritonitis.

\section{CONCLUSION}

Optimal dosing for vancomycin should consider both the pharmacokinetic (concentration in dialysis fluid and plasma), RKF, PD modality, and physicochemical factors (bioavailability, permeability) and pharmacodynamics (MIC and variability to the susceptibilities of the organism). Generally, vancomycin is given intraperitoneally during the long day dwell for patients on APD; this approach supports adequate equilibration during the absorption phase between dialysate and plasma to reach therapeutic levels. In addition, the impact of rapid cycling and RKF on the total body clearance has yet to be fully defined. With this in mind, TDM may be appropriate, however, there is yet to be an established protocol in PD patients with peritonitis. As the option to temporarily switch to CAPD in APD patients who develop peritonitis may not be convenient, the need for future research on the impact of the cycler on vancomycin clearance is imperative. Upcoming studies (NCT03685747) examining the pharmacokinetic of vancomycin will address some of the knowledge gaps associated with vancomycin pharmacokinetic in patients on APD. For the moment, clinicians should consider the bioavailability, dwell time, and institutional microbiological susceptibilities when dosing vancomycin in PD. Dedicated pharmacokinetic studies in adult and pediatric patients are needed to understand vancomycin disposition in PD patients on rapid-cycling modalities. The integrated use of TDM and MICs via dosing algorithms may help improve clinical outcome. 


\section{Conflict of Interests Disclosure}

359 No competing interests.

360 We have read and understood Peritoneal Dialysis International's policy on disclosing conflicts of interest

361 and declare that we have none. 


\section{References}

1. Salzer WL. Peritoneal dialysis-related peritonitis: Challenges and solutions. Int J Nephrol Renovasc Dis. 2018; 11:173-186.

2. Marsot A, Boulamery A, Bruguerolle B, Simon N. Vancomycin: A review of population pharmacokinetic analyses. Clin Pharmacokinet. 2012; 51:1-13.

3. Rybak M, Lomaestro B, Rotschafer JC, Moellering R, Jr., Craig W, Billeter M, et al. Therapeutic monitoring of vancomycin in adult patients: A consensus review of the american society of health-system pharmacists, the infectious diseases society of america, and the society of infectious diseases pharmacists. Am J Health Syst Pharm. 2009; 66:82-98.

4. Matzke GR, McGory RW, Halstenson CE, Keane WF. Pharmacokinetics of vancomycin in patients with various degrees of renal function. Antimicrob Agents Chemother. 1984; 25:433-7.

5. Matzke GR, Zhanel GG, Guay DR. Clinical pharmacokinetics of vancomycin. Clin Pharmacokinet. 1986; 11:257-82.

6. Tenover FC, Moellering RC, Jr. The rationale for revising the clinical and laboratory standards institute vancomycin minimal inhibitory concentration interpretive criteria for staphylococcus aureus. Clin Infect Dis. 2007; 44:1208-15.

7. Sakoulas G, Moise-Broder PA, Schentag J, Forrest A, Moellering RC, Jr., Eliopoulos GM. Relationship of mic and bactericidal activity to efficacy of vancomycin for treatment of methicillin-resistant staphylococcus aureus bacteremia. J Clin Microbiol. 2004; 42:2398-402.

8. Howden BP, Ward PB, Charles PG, Korman TM, Fuller A, du Cros P, et al. Treatment outcomes for serious infections caused by methicillin-resistant staphylococcus aureus with reduced vancomycin susceptibility. Clin Infect Dis. 2004; 38:521-8.

9. Moise-Broder PA, Forrest A, Birmingham MC, Schentag JJ. Pharmacodynamics of vancomycin and other antimicrobials in patients with staphylococcus aureus lower respiratory tract infections. Clin Pharmacokinet. 2004; 43:925-42.

10. Filippone EJ, Kraft WK, Farber JL. The nephrotoxicity of vancomycin. Clin Pharmacol Ther. 2017; 102:459-469.

11. Brophy DF, Sowinski KM, Kraus MA, Moe SM, Klaunig JE, Mueller BA. Small and middle molecular weight solute clearance in nocturnal intermittent peritoneal dialysis. Perit Dial Int. 1999; 19:534-9.

12. Manley HJ, Bailie GR, Frye RF, McGoldrick MD. Intravenous vancomycin pharmacokinetics in automated peritoneal dialysis patients. Perit Dial Int. 2001; 21:378-85.

13. Brouard RJ, Kapusnik JE, Gambertoglio JG, Schoenfeld PY, Sachdeva M, Freel K, et al. Teicoplanin pharmacokinetics and bioavailability during peritoneal dialysis. Clin Pharmacol Ther. 1989; 45:674-81. 
441

442

443

444

445

446

447

448

449

450

451

452

453

454

14. Morse GD, Apicella MA, Walshe JJ. Absorption of intraperitoneal antibiotics. Drug Intell Clin Pharm. 1988; 22:58-61.

15. Morse GD, Farolino DF, Apicella MA, Walshe JJ. Comparative study of intraperitoneal and intravenous vancomycin pharmacokinetics during continuous ambulatory peritoneal dialysis. Antimicrob Agents Chemother. 1987; 31:173-7.

16. Montanes Pauls B, Alminana MA, Casabo Alos VG. Vancomycin pharmacokinetics during continuous ambulatory peritoneal dialysis in patients with peritonitis. Eur J Pharm Sci. 2011; 43:212-6.

17. Blowey DL, Warady BA, Abdel-Rahman S, Frye RF, Manley HJ. Vancomycin disposition following intraperitoneal administration in children receiving peritoneal dialysis. Perit Dial Int. 2007; 27:79-85.

18. Rogge MC, Johnson CA, Zimmerman SW, Welling PG. Vancomycin disposition during continuous ambulatory peritoneal dialysis: A pharmacokinetic analysis of peritoneal drug transport. Antimicrob Agents Chemother. 1985; 27:578-82.

19. Bailie GR, Eisele G, Venezia RA, Yocum D, Hollister A. Prediction of serum vancomycin concentrations following intraperitoneal loading doses in continuous ambulatory peritoneal dialysis patients with peritonitis. Clin Pharmacokinet. 1992; 22:298-307.

20. Neal D, Bailie GR. Clearance from dialysate and equilibration of intraperitoneal vancomycin in continuous ambulatory peritoneal dialysis. Clin Pharmacokinet. 1990; 18:485-90.

21. Paton TW, Cornish WR, Manuel MA, Hardy BG. Drug therapy in patients undergoing peritoneal dialysis. Clinical pharmacokinetic considerations. Clin Pharmacokinet. 1985; 10:404-25.

22. Blevins RD, Halstenson CE, Salem NG, Matzke GR. Pharmacokinetics of vancomycin in patients undergoing continuous ambulatory peritoneal dialysis. Antimicrob Agents Chemother. 1984; 25:603-6.

23. Pancorbo S, Comty C. Peritoneal transport of vancomycin in 4 patients undergoing continuous ambulatory peritoneal dialysis. Nephron. 1982; 31:37-9.

24. Harford AM, Sica DA, Tartaglione T, Polk RE, Dalton HP, Poynor W. Vancomycin pharmacokinetics in continuous ambulatory peritoneal dialysis patients with peritonitis. Nephron. 1986; 43:217-22.

25. Bunke CM, Aronoff GR, Brier ME, Sloan RS, Luft FC. Vancomycin kinetics during continuous ambulatory peritoneal dialysis. Clin Pharmacol Ther. 1983; 34:631-7.

26. Whitby M, Edwards R, Aston E, Finch RG. Pharmacokinetics of single dose intravenous vancomycin in capd peritonitis. J Antimicrob Chemother. 1987; 19:351-7. 
27. Tobudic S, Matzneller P, Stoiser B, Wenisch JM, Zeitlinger M, Vychytil A, et al. Pharmacokinetics of intraperitoneal and intravenous fosfomycin in automated peritoneal dialysis patients without peritonitis. Antimicrob Agents Chemother. 2012; 56:3992-5.

28. Wiesholzer M, Pichler P, Reznicek G, Wimmer M, Kussmann M, Balcke P, et al. An open, randomized, single-center, crossover pharmacokinetic study of meropenem after intraperitoneal and intravenous administration in patients receiving automated peritoneal dialysis. Antimicrob Agents Chemother. 2016; 60:2790-7.

29. Li PK, Szeto CC, Piraino B, de Arteaga J, Fan S, Figueiredo AE, et al. Ispd peritonitis recommendations: 2016 update on prevention and treatment. Perit Dial Int. 2016; 36:481-508.

30. Cardone KE, Chen WZ, Grabe DW, Batzold A, Manley HJ, Lodise TP. Evaluation of the pharmacodynamic profile of commonly used intravenous vancomycin dosing schemes in patients on automated peritoneal dialysis. J Antimicrob Chemother. 2014; 69:1873-6.

31. Li PK, Szeto CC, Piraino B, Bernardini J, Figueiredo AE, Gupta A, et al. Peritoneal dialysis-related infections recommendations: 2010 update. Perit Dial Int. 2010; 30:393-423.

32. Blunden M, Zeitlin D, Ashman N, Fan SL. Single uk centre experience on the treatment of pd peritonitis--antibiotic levels and outcomes. Nephrol Dial Transplant. 2007; 22:1714-9.

33. Tosukhowong T, Eiam-Ong S, Thamutok K, Wittayalertpanya S, Na Ayudhya DP. Pharmacokinetics of intraperitoneal cefazolin and gentamicin in empiric therapy of peritonitis in continuous ambulatory peritoneal dialysis patients. Perit Dial Int. 2001; 21:587-94.

34. Whitty R, Bargman JM, Kiss A, Dresser L, Lui P. Residual kidney function and peritoneal dialysisassociated peritonitis treatment outcomes. Clin J Am Soc Nephrol. 2017; 12:2016-2022.

35. Fish R, Nipah R, Jones C, Finney H, Fan SL. Intraperitoneal vancomycin concentrations during peritoneal dialysis-associated peritonitis: Correlation with serum levels. Perit Dial Int. 2012; 32:332-8.

36. Mulhern JG, Braden GL, O'Shea MH, Madden RL, Lipkowitz GS, Germain MJ. Trough serum vancomycin levels predict the relapse of gram-positive peritonitis in peritoneal dialysis patients. Am J Kidney Dis. 1995; 25:611-5.

37. Stevenson S, Tang W, Cho Y, Mudge DW, Hawley CM, Badve SV, et al. The role of monitoring vancomycin levels in patients with peritoneal dialysis-associated peritonitis. Perit Dial Int. 2015; $35: 222-8$.

38. Brown J, Altmann P, Cunningham J, Shaw E, Marsh F. Pharmacokinetics of once daily intraperitoneal aztreonam and vancomycin in the treatment of capd peritonitis. J Antimicrob Chemother. 1990; 25:141-7.

39. Gendeh BS, Gibb AG, Aziz NS, Kong N, Zahir ZM. Vancomycin administration in continuous ambulatory peritoneal dialysis: The risk of ototoxicity. Otolaryngol Head Neck Surg. 1998; 118:551-8. 
Table 1. Vancomycin absorption parameters in adult and pediatric non-infected and PD-related peritonitis patients on peritoneal dialysis.

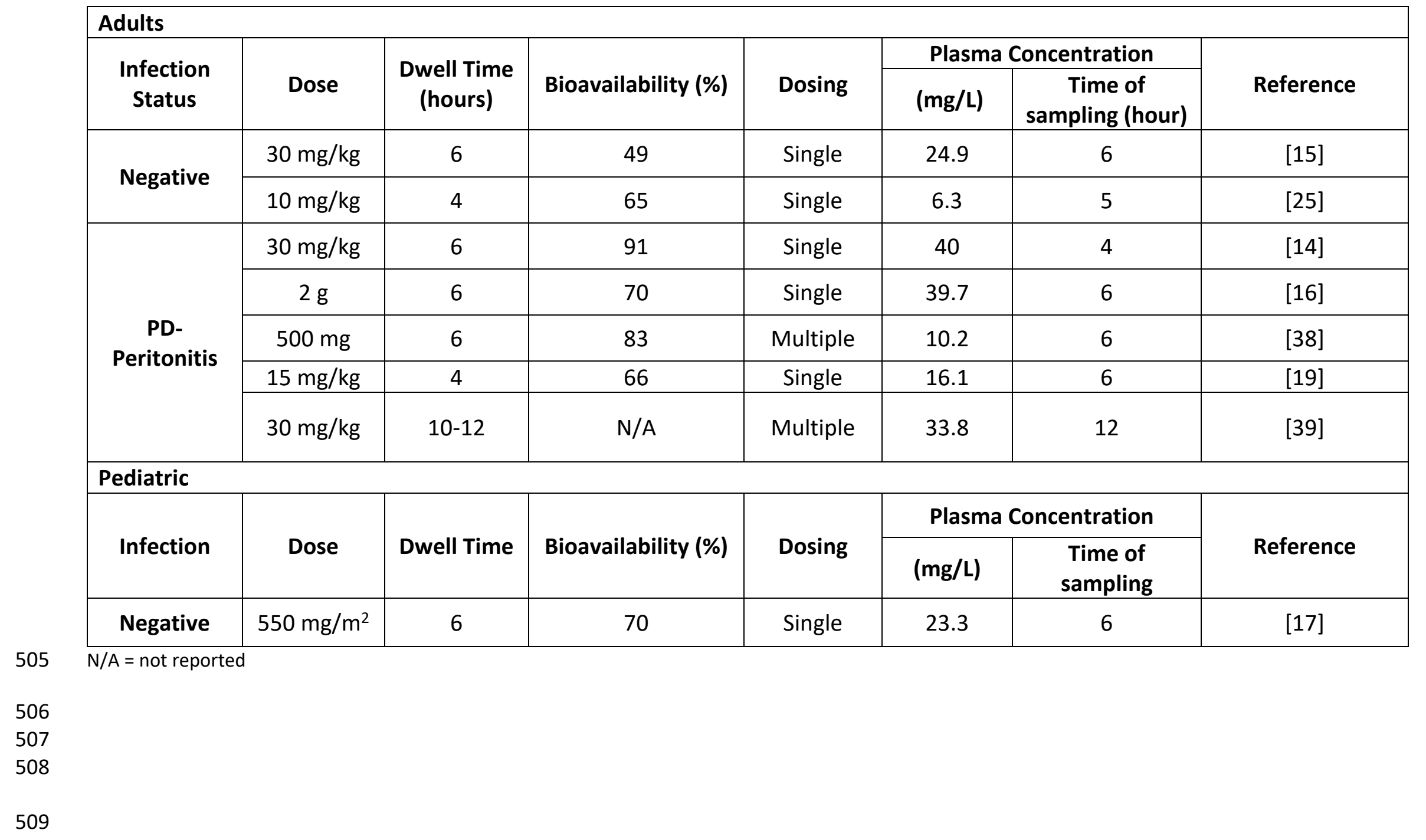




\begin{tabular}{|c|c|c|c|c|c|c|c|c|}
\hline \multicolumn{9}{|l|}{ Adults } \\
\hline \multirow{2}{*}{ Modality } & \multirow{2}{*}{ Infection Status } & \multirow{2}{*}{ Route } & \multirow{2}{*}{$V_{d}(L / k g)$} & \multirow{2}{*}{$\begin{array}{l}\text { Plasma Half- } \\
\text { life (hours) }\end{array}$} & \multicolumn{3}{|c|}{ Clearance (mL/min) } & \multirow{2}{*}{ Reference } \\
\hline & & & & & Total & Dialytic & Renal & \\
\hline \multirow{6}{*}{ CAPD } & \multirow[t]{2}{*}{ Negative } & IP & 0.56 & 111 & 5 & 1.2 & N/A & {$[15]$} \\
\hline & & IV & 0.73 & 92 & 6.4 & 1.4 & 0.65 & {$[22]$} \\
\hline & \multirow{4}{*}{ PD-Peritonitis } & IP & 0.61 & N/A & N/A & 15.7 & N/A & [19] \\
\hline & & IP & 0.87 & N/A & 8.5 & 12.2 & N/A & [20] \\
\hline & & IV & 0.55 & 104 & 4.1 & 3.8 & N/A & [24] \\
\hline & & IV & 1.1 & 115 & 7.2 & 1.4 & N/A & [26] \\
\hline APD & Negative & IV & 0.4 & $11.6 / 62.8^{a}$ & 7.4 & 2.1 & 1.7 & [12] \\
\hline \multicolumn{9}{|l|}{ Pediatric } \\
\hline \multirow{2}{*}{ Modality } & \multirow{2}{*}{ Infection } & \multirow{2}{*}{ Route } & \multirow{2}{*}{$V_{d}(L / k g)$} & \multirow{2}{*}{ Plasma } & \multicolumn{3}{|c|}{ Clearance $\left(\mathrm{mL} / \mathrm{min} / 1.73 \mathrm{~m}^{2}\right)$} & \multirow{2}{*}{ Reference } \\
\hline & & & & & Total & Dialytic & Renal & \\
\hline CAPD & \multirow{2}{*}{ Negative } & \multirow{2}{*}{ IP } & \multirow{2}{*}{0.48} & \multirow{2}{*}{25} & 10.7 & 2.5 & \multirow{2}{*}{1.4} & \multirow{2}{*}{ [17] } \\
\hline APD & & & & & 14.9 & 3.1 & & \\
\hline
\end{tabular}


Table 3. Pharmacokinetic/pharmacodynamic factors for TDM consideration between CAPD and APD vancomycin regimens.

\begin{tabular}{|c|c|c|c|}
\hline Pharmacokinetic/pharmacodynamics & PD components & CAPD & APD \\
\hline \multirow{2}{*}{ Absorption } & Dwell time & $\downarrow$ Bioavailability & 个 Bioavailability \\
\hline & Dosing route (IP vs. IV) & \multicolumn{2}{|c|}{ Same } \\
\hline \multirow{5}{*}{ Distribution } & $\begin{array}{c}\text { Permeability (Peritonitis vs. } \\
\text { non-peritonitis) }\end{array}$ & \multirow{5}{*}{\multicolumn{2}{|c|}{ Same }} \\
\hline & Diffusion & & \\
\hline & Protein binding & & \\
\hline & Surface area & & \\
\hline & Vascularity & & \\
\hline \multirow{4}{*}{ Elimination } & Dosing route (IP vs. IV) & \multicolumn{2}{|c|}{ RKF- Drives variation in systemic circulation } \\
\hline & Body size \& Dialysate volume & \multicolumn{2}{|c|}{ Same- Patient dependent } \\
\hline & Dwell time & 个 Clearance & $\downarrow$ Clearance \\
\hline & $\begin{array}{c}\text { Number of non-antibiotic } \\
\text { exchanges }\end{array}$ & $\downarrow$ Clearance & $\uparrow$ Clearance \\
\hline Pharmacodynamics & MIC/AUC & \multicolumn{2}{|c|}{ Same- Susceptibility report } \\
\hline
\end{tabular}


Table 4. Proposal for critical research areas to optimize vancomycin therapy in peritoneal dialysis.

Proposal for Critical Research Areas of Needed Research for Vancomycin Therapy in Peritoneal Dialysis

- Effect of APD on peritoneal and plasma levels during rapid cycles

- Peak concentration following absorption from the long-dwell

- Optimal trough concentrations associated with improved clinical outcomes and the timing of trough monitoring specific for the peritoneal dialysis population

- Dosing regimen to achieve optimal trough concentrations

- Effect of residual kidney function on vancomycin disposition and its implications on dosing

- Factors affecting non-renal and non-dialytic clearance of vancomycin

- Determining appropriate clinical plasma sampling time points 
530

531

532

533

534

535

536

537

538

539

540

541

542

\section{Vancomycin Absorption \& Distribution}
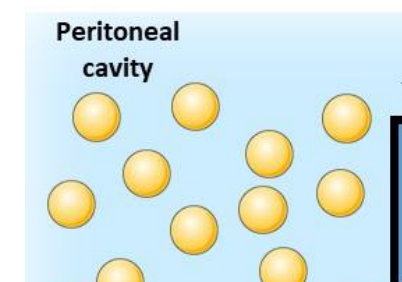

Dialysate Properties

- $\uparrow$ Dwell time $=\uparrow$ Extent absorbed

- Dialysate and drug compatibility

- Volume

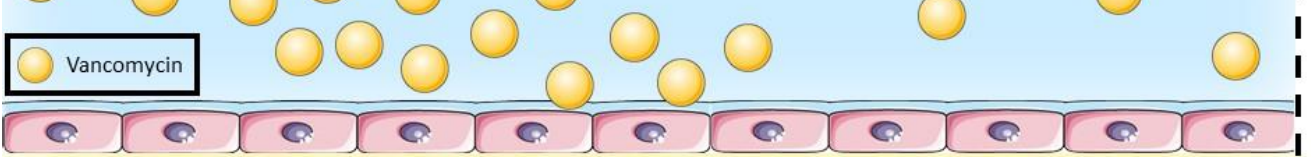

$$
\begin{aligned}
& \text { Peritoneum Properties } \\
& \text { Permeability } \\
& \text { Peritoneal } \\
& \text { Capillaries }
\end{aligned}
$$

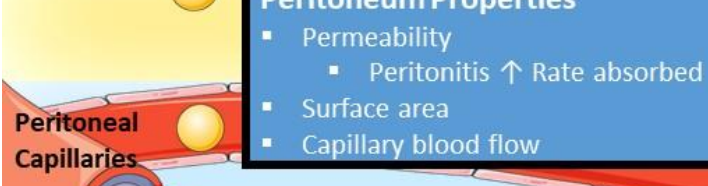

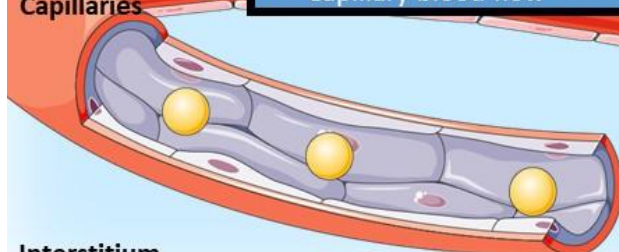

Interstitium
Dwell Time (Hours)

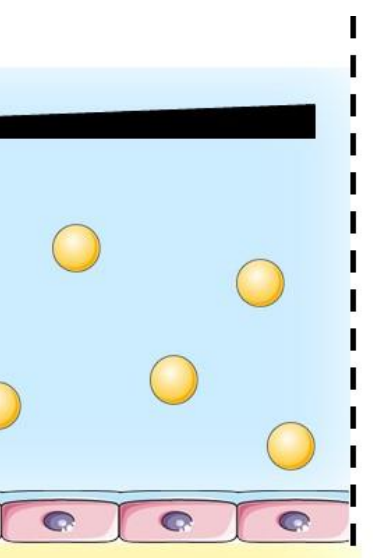

I

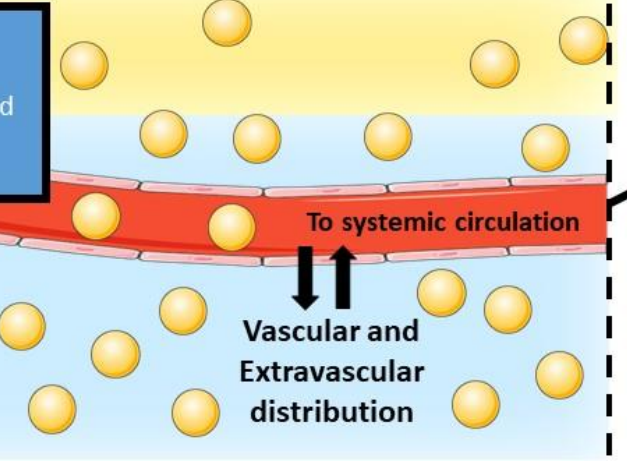

Clearance

Residual kidney function

Total Body Clearance

- Residual kidney function

- Peritoneal dialysis

- Other (e.g. Iymphatics)

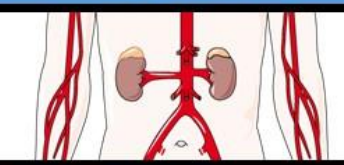

Dialytic Clearance

- Clearance from CAPD or APD

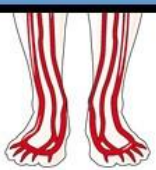

Figure 1. Illustration of vancomycin absorption, distribution and elimination following an intraperitoneal dose.

Increasing the dwell time enhances vancomycin bioavailability. Peritoneum and dialysate properties should be considered as these both affect the rate and extent of absorption following an intraperitoneal dose. Following dosing and an appreciable dwell time, vancomycin is eliminated by PD, renal, and non-renal sources. These processes make up the total body clearance of vancomycin.

This illustration is a derivative of "Simple squamous epithelium", "Arteries", "Arterial circulation" and "Bubble" by Servier Medical Art (https://smart.servier.com/) under the Creative Commons License (CC BY 3.0). 
- Gram-positive coverage

- Dose at $15-20 \mathrm{mg} / \mathrm{kg}$

- Allow to dwell for $10-15$ hours

Does the patient have residual kidney function with a creatinine clearance YES $>5 \mathrm{~mL} / \mathrm{min}$ ?

559 Figure 2. Proposed vancomycin dosing and monitoring algorithm in patients on automated peritoneal dialysis. 